\title{
Micromachined Fiber Optical Sensor for In Vivo Measurement of Optical Properties of Human Skin
}

\author{
Alejandro Garcia-Uribe, Karthik Chinna Balareddy, Jun Zou, Member, IEEE, and Lihong V. Wang, Fellow, IEEE
}

\begin{abstract}
In this paper, we present the design, fabrication, and testing of a new micromachined fiber optic sensor probe to conduct oblique incidence diffuse reflectance spectrometry (OIDRS) for in vivo estimation of optical properties of human skins. The probe consists of three source fibers, two linear array of collection fibers, and four micromachined positioning devices for accurate alignment of the fibers. Micromachining plays a significant role in the probe development by enabling device miniaturization, low-cost fabrication, and precise assembly. The new probe has been successfully used to estimate the absorption and scattering coefficient spectra of skin with an optical spectrum between 455 and $765 \mathrm{~nm}$.
\end{abstract}

Index Terms-Absorption coefficient, diffuse reflectance, micromachined optical probe, scattering coefficient.

\section{INTRODUCTION}

$\mathbf{T}$ HE human skin is a heterogeneous media and its optical properties can vary significantly even between close sites. Recent study has suggested the close relationship between the stage of skin diseases (e.g., various skin cancers) and the optical (absorption and scattering) properties of the affected skin area [1]-[4]. Thus, the development of in vivo methods to accurately characterize localized optical properties of human skins will greatly assist in the diagnosis and even treatment of its pathologies [5]-[11]. Oblique incidence diffuse reflectance spectrometry (OIDRS) is an optical method capable of quantifying both bulk absorption and scattering optical properties of heterogeneous media (e.g., human skins) by measuring the diffuse reflectance of the media. Previous studies have shown the use of oblique incidence diffuse reflectance spectroscopy to statistically differentiate skin carcinoma from actinic and seborrheic keratoses [12]. The handmade probe use in [12] was a limiting factor for a reliable estimate of absorption and reduced scattering coefficients. New optical sensor probes are necessary to ensure a rapid and reliable measurement of the diffuse reflectance spectra of the targeted area especially in a clinical environment. In this paper, we report the development of a new OIDRS probe fabricated with micromachining technology. The precision and mass production capability of micromachining ensures the needed performance, as well as the compact size and low-cost fabrication of the probe. Using this probe, the op-

\footnotetext{
Manuscript received February 3, 2008; accepted March 13, 2008. This work was supported by the National Institute of Health under Grant R01 CA106728. The associate editor coordinating the review of this manuscript and approving it for publication was Prof. Okyay Kaynak.

A. Garcia-Uribe, K. C. Balareddy, and J. Zou are with the Department of Electrical and Computer Engineering, Texas A\&M University, College Station, TX 77843 USA (e-mail: aguribe@ @amu.edu).

L. V. Wang is with the Department of Biomedical Engineering, Washington University in St. Louis, St. Louis, MO 63130 USA.

Digital Object Identifier 10.1109/JSEN.2008.2003306
}

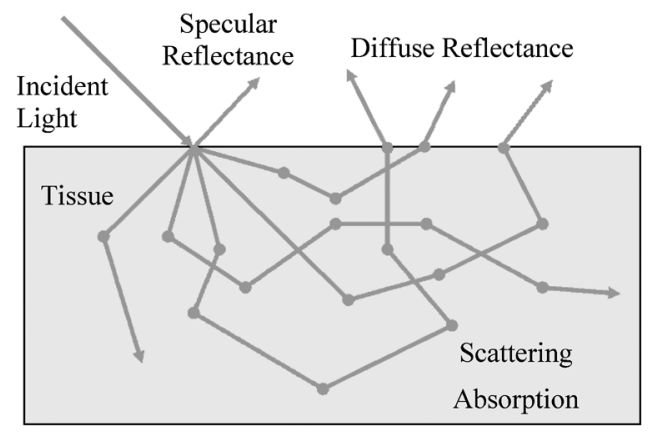

Fig. 1. Light interaction in a scattering and absorbing media.

tical absorption and scattering properties of human skins have been successfully characterized.

\section{PRINCIPLE OF OIDRS}

As shown in Fig. 1, when light is incident on the surface of a heterogeneous media (e.g., human skin), part of the incident light will be directly reflected (specular reflectance) and the remainder will transmit into and will interact with the media. After undergoing multiple times of scattering and absorption, part of the transmitted light will be "turned" back and will escape from the surface of the media, which forms the diffuse reflectance.

According to the diffusion theory, the spatially resolved steady-state diffuse reflectance at certain wavelength $\left(R_{d}(x)\right)$ for oblique incidence can be calculated using a modified two-source approximation with one positive source located below the sample surface and one negative source located above the sample surface (Fig. 2) [13], [14], which can be determined by

$$
\begin{aligned}
R_{d}(x)=\frac{1}{4 \pi}[ & \frac{\Delta z\left(1+\mu_{\mathrm{eff}} \rho_{1}\right) \exp \left(-\mu_{\mathrm{eff}} \rho_{1}\right)}{\rho_{1}^{3}} \\
& \left.+\frac{\left(\Delta z+2 z_{b}\right)\left(1+\mu_{\mathrm{eff}} \rho_{2}\right) \exp \left(-\mu_{\mathrm{eff}} \rho_{2}\right)}{\rho_{2}^{3}}\right]
\end{aligned}
$$

where $\mu_{\mathrm{eff}}$ is the effective attenuation coefficient, $\rho_{1}$ and $\rho_{2}$ are the distances between the two isotropic point sources (one positive source located below the media surface and one negative source located above the media surface) and the observation point on the surface of the media, $\Delta z$ is the distance between the virtual boundary and the media depth, and $\mathrm{z}_{\mathrm{b}}$ is the distance between the virtual boundary and the surface of the media. The absorption coefficient $\left(\mu_{a}\right)$ and reduced scattering coefficient $\left(\mu_{s}^{\prime}\right)$ can be determined as [13]

$$
\mu_{a}=\frac{\mu_{\mathrm{eff}}^{2} \Delta x}{3 \sin \left(\alpha_{t}\right)}
$$




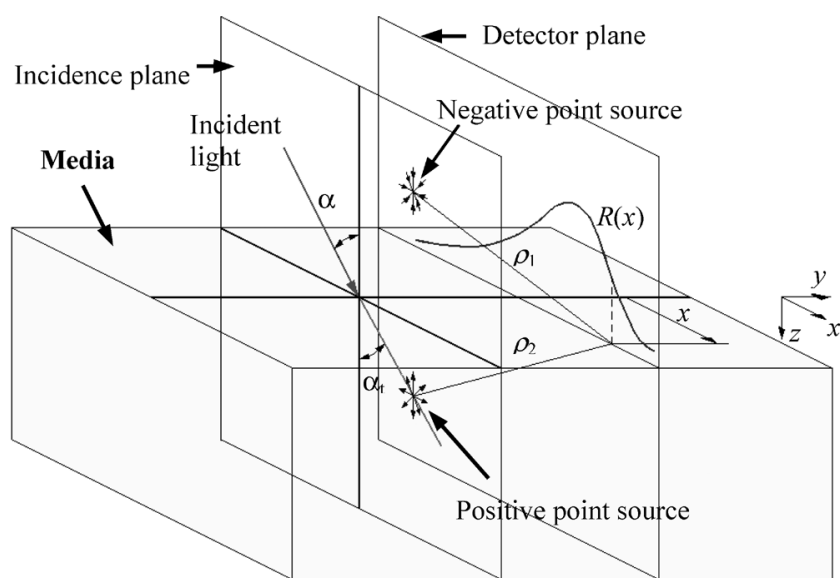

(a)

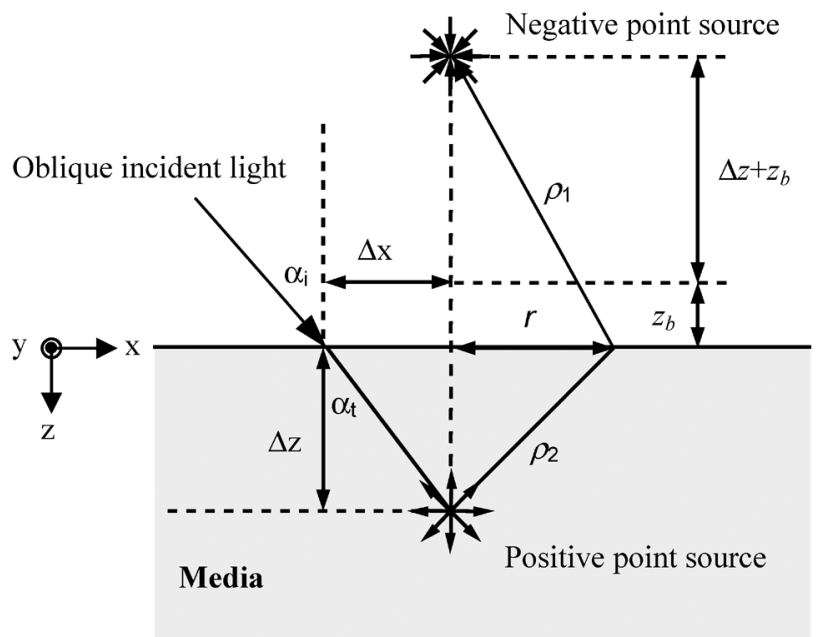

(b)

Fig. 2. (a) Schematic of the modified two-source approximation of oblique incidence based on diffusion theory. (b) Schematic of the incidence plane.

$$
\mu_{s}^{\prime}=\frac{\sin \left(\alpha_{t}\right)}{\Delta x}-0.35 \mu_{a}
$$

where $\Delta x$ is the shift of the point sources in the $x$ direction and $\alpha_{t}$ is the angle of light transmission into the media. Therefore, by measuring the spatially resolved diffuse reflectance $\left(R_{d}(x)\right)$, $\Delta x, \alpha_{t}$, and $\mu_{\text {eff }}$ can be experimentally determined and then $\mu_{a}$ and $\mu_{s}^{\prime}$ can be estimated. It should be noted that the diffusion equation assumes that the reduced scattering coefficient is much larger than the absorption. The source and the detector must also be separated in space so that the light solely consists of diffuse reflectance when it reaches the detector.

\section{SENSOR PROBE DESIGN}

To conduct OIDRS measurement on human skins, we have developed a handheld fiber optical sensor probe to facilitate convenient and robust data collection in a clinical environment (Fig. 3). The sensor probe consists of three source fibers and two linear arrays of collection fibers for capturing the spatial distribution of diffuse reflectance $\left(R_{d}(x)\right)$. The effective probe testing area is limited to $2 \times 2 \mathrm{~m}^{2}$ to ensure that the measured area does not include the surrounding normal skin even for the smallest skin lesions (usually around $3 \times 3 \mathrm{~mm}^{2}$ ). Among the

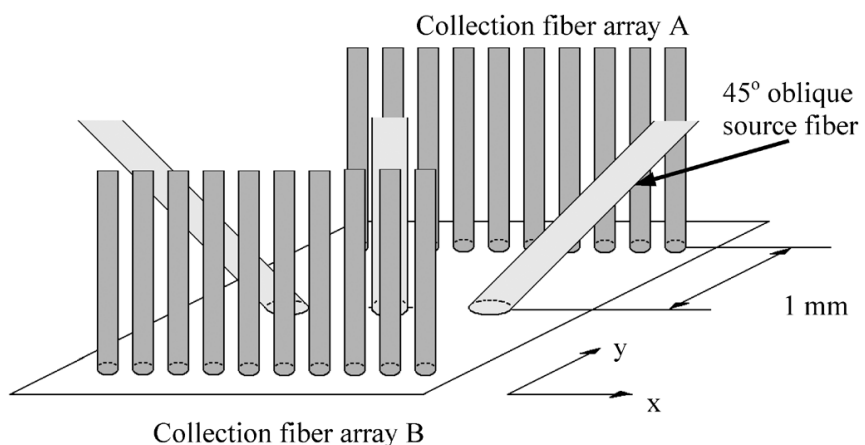

Fig. 3. Schematic design of the OIDRS probe.

three source fibers, two are used for oblique incidence (delivering light onto the skin surface at an oblique angle $(\alpha)$ of $45^{\circ}$. Because the OIDRS measurement is usually performed in a dark environment to reduce the effect of the background light, the center normal incidence source fiber is used to illuminate area of interest on the skin to ensure the accurate placement of the sensor probe. Although only one oblique incidence fiber and one linear array of collection fibers are necessary for an OIDRS measurement, two oblique incidence fibers and two arrays of collection fibers are used for multiple data collections from the same location on the skin to ensure a reliable and robust measurement.

For the source fibers, optical fibers with a diameter of $200 \mu \mathrm{m}$ are used to provide enough incident light to ensure high signal-to-noise ratio (SNR) of the measurement, especially for dark-color skins. For the collection fibers, each of the two linear arrays consists of ten fibers with a diameter of $100 \mu \mathrm{m}$ and a center-to-center pitch of $200 \mu \mathrm{m}$. Based on our previous simulation, at least ten data points are needed for a span of $2 \mathrm{~mm}$ to achieve a good spatial resolution for the estimation of the optical absorption and scattering properties with good accuracy. The $100-\mu \mathrm{m}$ diameter of the collection fibers is expected to provide satisfactory SNR for the measurement of diffuse reflectance. The use of smaller collection fibers will result in lower SNR and the use of bigger collection fibers would unnecessarily increase the size of the probe or lower the spatial resolution of data sampling. The two collection fiber arrays are separate from the incidence fibers by $1 \mathrm{~mm}$. This is because the estimation of the absorption and scattering coefficients from the measured diffuse reflectance is based on the diffusion theory, which is accurate only when the distance between the detector and the source is greater than one mean free path $L_{\mathrm{t}}^{\prime}=1 /\left(\mu_{a}+\mu_{s}^{\prime}\right)$ (typically, about $0.1 \mathrm{~cm}$ for biological tissues) [15]. Although larger separation could be used, it will unnecessarily increase the overall size of the sensor probe.

\section{SEnsor Probe Fabrication}

To ensure the accuracy of the OIDRS measurements, the source fibers and collection fibers need to be precisely aligned with respect to each other and fixed in their own positions. This can be achieved with a compact mechanical positioning device. However, due to the small size and dense arrangement of the fibers, it is very difficult and costly to fabricate the mechanical positioning device using conventional machining methods. To 


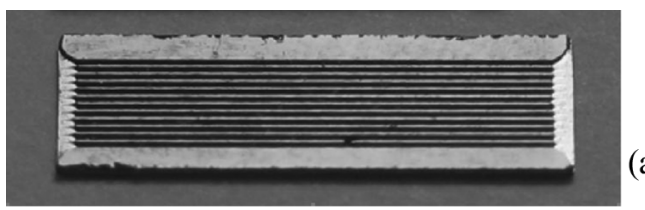

(a)

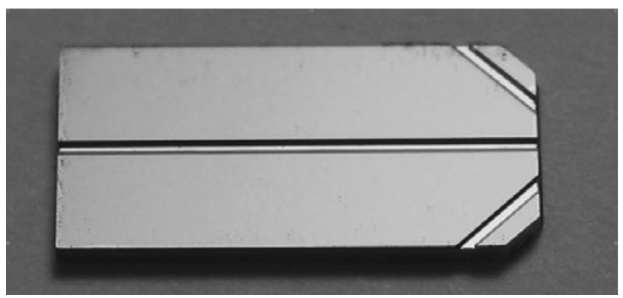

(b)

Fig. 4. Micromachined positioning devices for alignment and assembly of optical fibers in the OIDRS probe: (a) for the collection fibers; (b) for the incident fibers.

address this issue, we have developed straightforward micromachining processes to achieve successful fabrication of the positioning devices in an efficient and low-cost manner.

To achieve accurate alignment of the collection fibers, two micromachined positioning devices were fabricated. Each positioning device consists of a silicon substrate with a linear array of V-grooves created with silicon bulk etching [Fig. 4(a)]. When an optical fiber (with cylindrical cross section) is placed in a V-groove, the center axis of the optical fiber can "automatically" align with the symmetric plane of the V-groove. Thus, the accurate positioning of each collection fiber in the array can be readily achieved to ensure reliable and uniform performance of the sensor probe. To fabricate the positioning device, silicon nitride was deposited on a $\{100\}$ silicon wafer. Photolithography and reactive ion etching was conducted to pattern the silicon nitride layer, which serves as a hard mask for silicon bulk etching. Silicon bulking etching was performed in potassium hydroxide solution to form the V-grooves $(130 \mu \mathrm{m}$ wide and $200 \mu \mathrm{m}$ apart).

For aligning the source fibers, two other micromachined positioning devices were fabricated. Because the need of guiding structures for the oblique incidence fibers $\left(45^{\circ}\right)$ precludes the fabrication of V-grooves with silicon bulk etching, SU-8 resist (MicroChem, Newton, MA) was used for the fabrication of the guiding structures on a silicon substrate [Fig. 4(b)]. SU-8 is preferable for this process as it can be directly used to form structures over $100 \mu \mathrm{m}$ in thickness, which results in a very simple and low-cost fabrication process. To fabricate the SU-8 guiding structure, a silicon substrate is first cleaned and backed at $200{ }^{\circ} \mathrm{C}$ for $5 \mathrm{~min}$. SU-8 100 resist was spun on the cleaned silicon substrate at calibrated spinning rate for $40 \mathrm{~s}$ to reach a final thickness of about $100 \mu \mathrm{m}$. A soft bake was conducted at $65^{\circ} \mathrm{C}$ for $10 \mathrm{~min}$ and then at $95^{\circ} \mathrm{C}$ for $30 \mathrm{~min}$, which was followed by an ultraviolet (UV) exposure of a dosage of about $520 \mathrm{~mJ} / \mathrm{cm}^{2}$. After the exposure, the wafer was baked at $65{ }^{\circ} \mathrm{C}$ for $1 \mathrm{~min}$ and then at $95{ }^{\circ} \mathrm{C}$ for 10 min to selectively cross link the exposed part of the SU-8 film. The development of exposed SU-8 film was conducted for a few minutes until unexposed region was completely removed. During the SU-8 processing, a slow temperature ramping was used to minimize the internal stress buildup and also the resulting crack formation within the SU-8

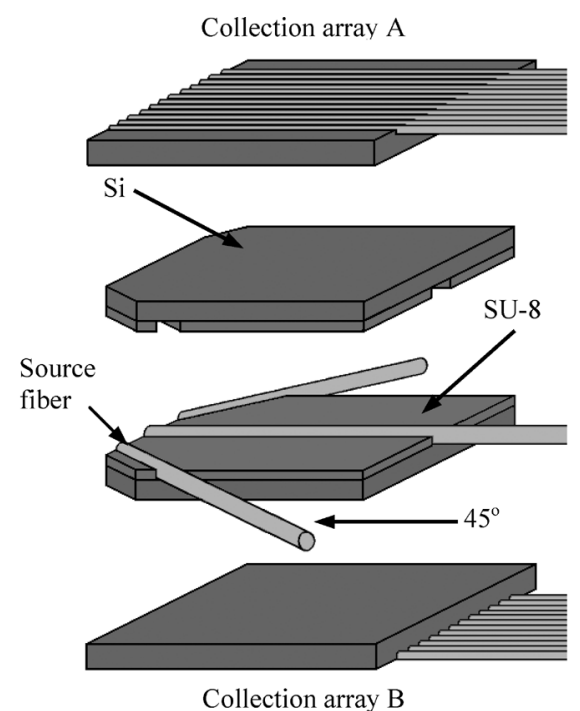

(a)

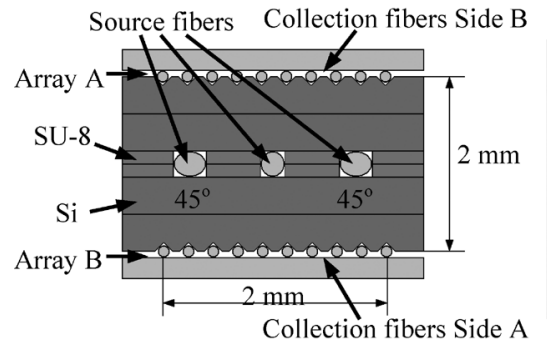

(b)

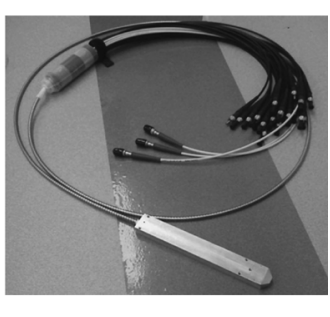

(c)
Fig. 5. (a) Probe assembly. (b) Schematic of the probe front view. (c) Complete probe.

film, which would significantly reduced the mechanical strength and stability of the guiding structures.

After the fabrication of the micropositioning devices was complete, the entire OIDRS sensor probe was assembled. First, both the source and collection fibers were fixed into their own guiding structures [Fig. 5(a)]. Because the thickness of the SU-8 guiding structure is $100 \mu \mathrm{m}$, two positioning devices were placed face-to-face to accommodate the source fibers (with a diameter of $200 \mu \mathrm{m}$ ). For the collection fibers, one positioning device and one cover substrate were used to hold them in place. After all the fibers were assembled, the positioning devices were stacked and glued together with epoxy [Fig. 5(b)]. Because the thickness of the silicon wafer used for the fabrication of the positioning devices is around $500 \mu \mathrm{m}$, the required 1-mm spacing between the source and collection fibers were readily obtained. To improve the efficiency of incidence and collection of the fibers, the head of the assembled probe was polished with sand papers. During the polishing, care was taken to avoid any possible damage to the fibers. Finally, the assembled probe was placed in an aluminum probe holder to facilitate OIDRS testing [Fig. 5(c)].

\section{OIDRS SYSTEM}

To conduct OIDRS measurement, we have built a complete experimental setup to interface with the developed sensor probe to achieve automated optical incidence control, data collection, and analysis (Fig. 6). It consists of white light source (halogen 


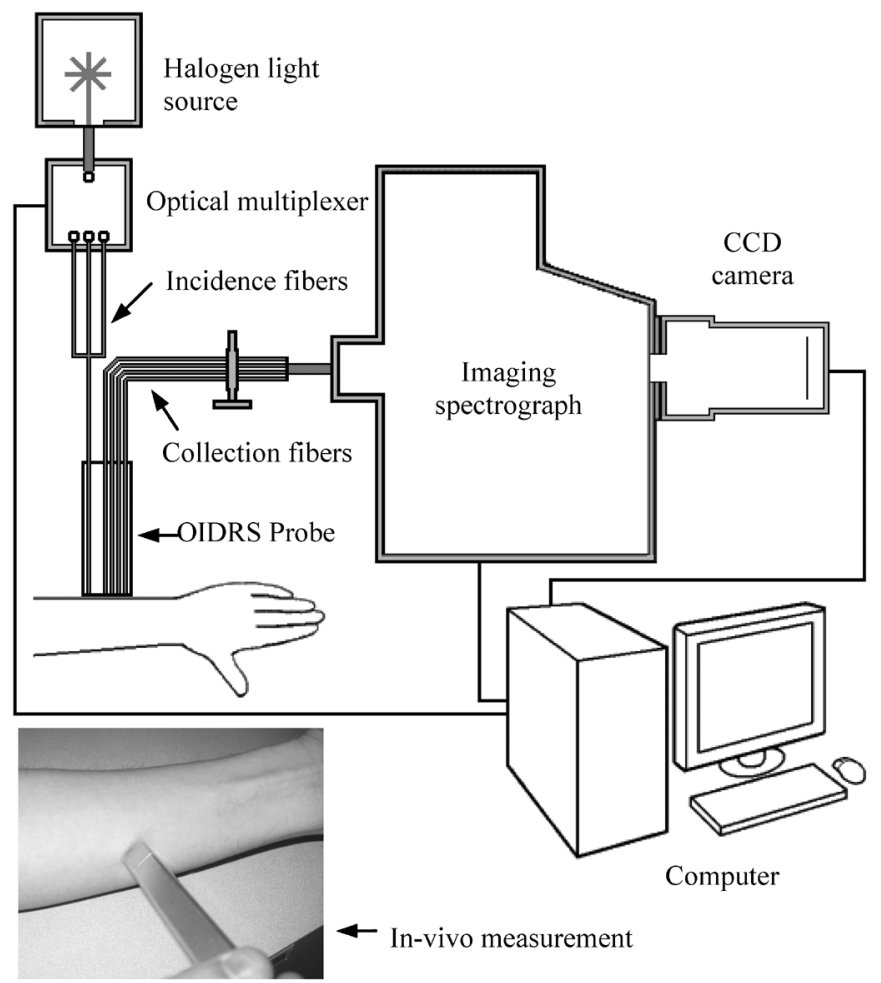

Fig. 6. Schematic of the OIDRS system.

lamp) for multiple-wavelength measurement, multiplexer, imaging spectrograph, charge-coupled device (CCD) camera, and personal computer. Before an OIDRS measurement is conducted, the three source fibers of the sensor probe are connected to the output of the light source via subminiature version A (SMA) connectors. The proximal end of each collection fiber is fitted with SMA 905 connectors and then connected to the input of the spectrograph through a custom-made interface. The optical multiplexer allows the light delivery, to the area of interest, through only one source fiber at a time. After the sensor probe gets into contact with the skin, white light is delivered through one of the source fibers and the diffuse reflectance is then captured by the collection fibers. The collection fibers are coupled with the imaging spectrograph that generates an optical spectrum for each fiber. The CCD camera collects the spectral images from the wavelength range of 455 to $765 \mathrm{~nm}$. The spectral images represent the steady-state diffuse reflectance spectra from each collection fiber, which are stored in the computer for further analysis. This system is capable of capturing one frame of spectral image in a fraction of a second.

Before the actual skin test was conducted, the experimental setup was calibrated and validated using a liquid reference solution (phantom) consisting of polystyrene microspheres as scattering elements and trypan blue as absorber [14]. The absorption coefficient spectra of trypan blue were measured by collimated transmission before mixing it with the polystyrene microspheres. The reduced scattering coefficient of the microspheres was calculated using Mie theory [16]. The "expected values" of the absorption and reduced scattering coefficients of the liquid reference solution can be vary by controlling the concentration of absorbing and scattering chemicals.

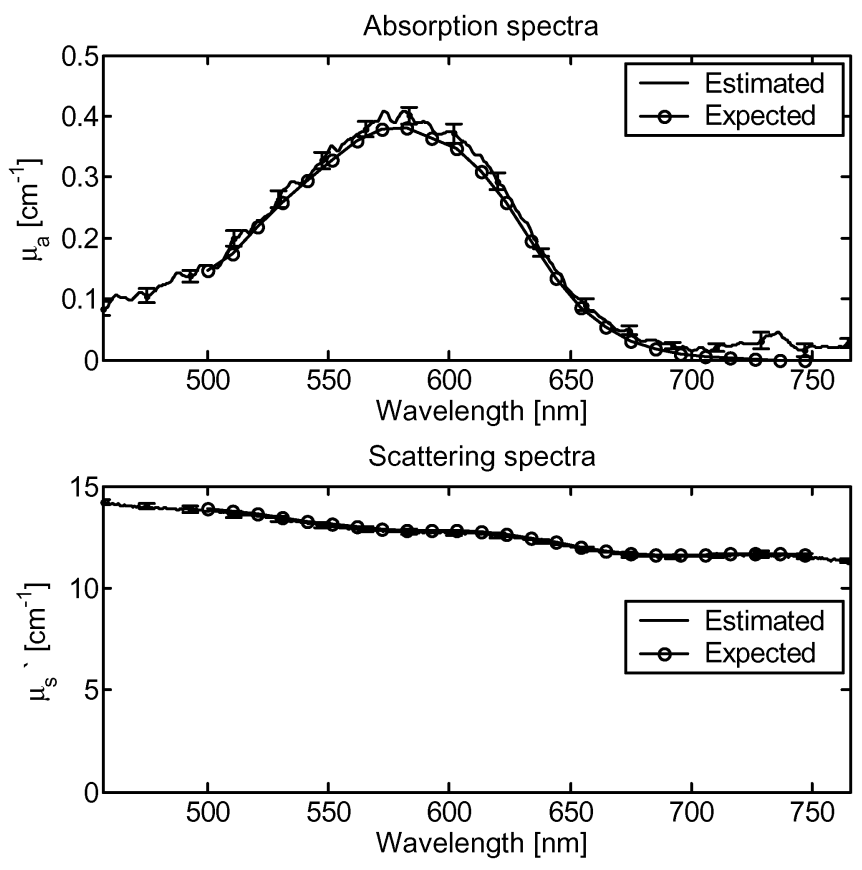

Fig. 7. Expected and estimated absorption and reduced scattering spectra of a liquid reference solution.

To conduct the calibration, the senor probe was placed on the surface of the reference solution. The probe was rotated to four different angles with respect to an arbitrary reference point, and the diffuse reflectance was recorded each time. The absorption and reduced scattering spectra were extracted for each diffuse reflectance measurement and averaged to obtain the "estimated values." The system was calibrated by measuring several optical reference phantoms. Each detector is compensated by a factor that matches the "estimated" diffuse reflectance to their expected values. The probe is then validated by estimating the optical properties of a deferent set of optical reference phantom (Fig. 7). After the calibration, the probe will be ready for the real measurements.

\section{IN VIVO MEASUREMENTS}

The OIDRS system is used to collect the steady-state spatially resolved diffuse reflectance spectra $R_{d}(x, \lambda)$ from human skin on the arm (Fig. 8). The main absorbers of human skin are hemoglobin and melanin. The extinction coefficients of melanin oxy-hemoglobin and deoxy-hemoglobin are shown in Fig. 9.

The extinction coefficient is the absorbance (of light) per unit path length and per unit of concentration $\left(\mathrm{cm}^{-1} \mathrm{mMoles}^{-1}\right)$. The lower reflectance points at the wavelengths 550 and $575 \mathrm{~nm}$ correspond to local stronger absorption caused by oxy-hemoglobin [17].

The absorption and scattering coefficients are calculated independently for each wavelength $\lambda$, using the corresponding diffuse reflectance along the $x$-axis. An example of the estimated absorption coefficient spectra $\mu_{a}(\lambda)$ and scattering coefficient spectra $\mu_{s}^{\prime}(\lambda)$ are shown in Fig. 10. The optical properties of the skin can differ largely depending on race, age, and location on the body [18]. Our results match closely with those obtained 


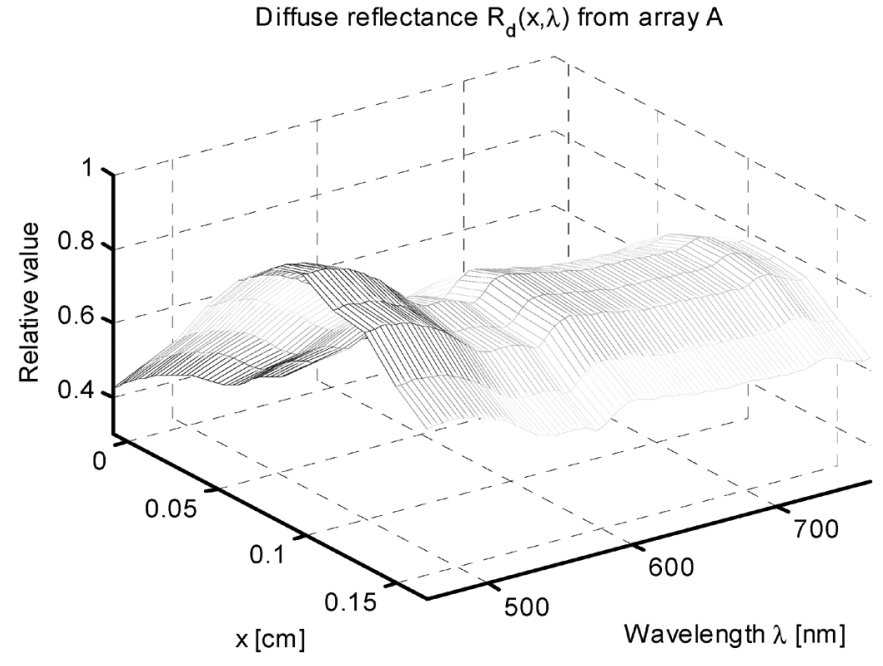

Fig. 8. Sample spatio-spectra diffuse reflectance data collected from human skin.

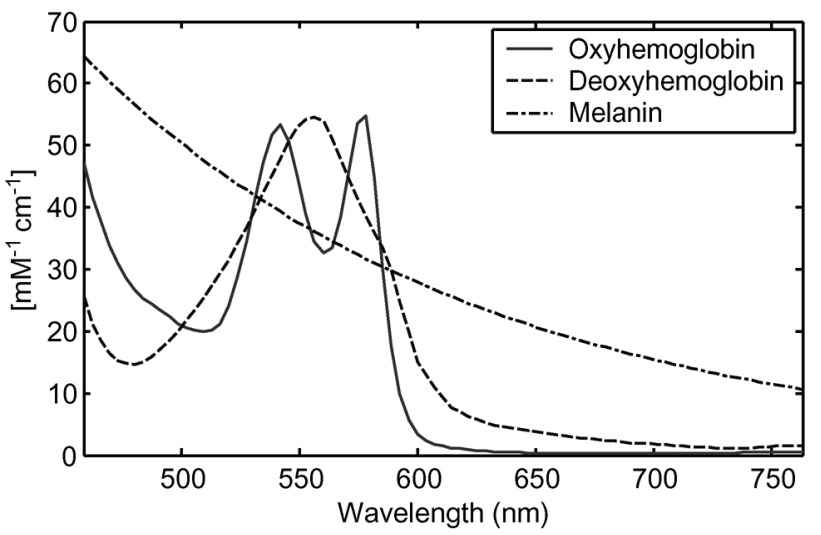

Fig. 9. Extinction coefficient of oxyhemoglobin, deoxyhemoglobin and melanin.
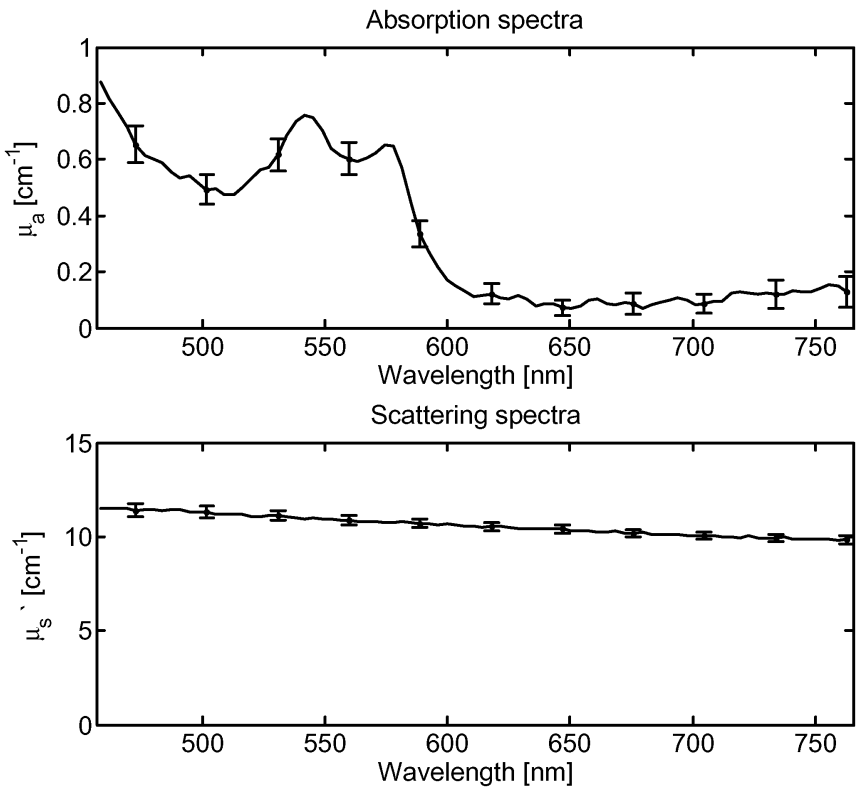

Fig. 10. Example of the estimated absorption and scattering coefficient from human skin. previously in ex vivo measurements presented in [19] and [20] and the in vivo measurements presented in [18] and [21].

The absorption coefficient can be used to estimate important physiological parameters related to the disease state such as the concentration of oxy-hemoglobin, deoxy-hemoglobin and its oxygen saturation $\left(\mathrm{StO}_{2}\right)$ [22]. For example, the absorption spectra $\mu_{a}(\lambda)$ is related to the concentration of the absorbers by

$\mu_{a}(\lambda)=\varepsilon_{\text {oxy }}(\lambda) C_{\text {oxy }}+\varepsilon_{\text {deoxy }}(\lambda) C_{\text {deoxy }}+\varepsilon_{\text {mel }}(\lambda) C_{\text {mel }}+\mu_{\text {abg }}$

where $\varepsilon_{\text {oxy }}(\lambda), \varepsilon_{\text {deoxy }}(\lambda)$, and $\varepsilon_{\text {mel }}(\lambda)$ are the known extinction coefficients of oxy-hemoglobin, deoxy-hemoglobin, and melanin at certain wavelength $(\lambda), C_{\text {oxy }}, C_{\text {deoxy }}$, and $C_{\text {mel }}$ are the concentrations (mMoles) of oxy-hemoglobin, deoxy-hemoglobin, and melanin, and $\mu_{\mathrm{abg}}$ is the absorption caused by other local tissue components. The oxygen saturation $\left(\mathrm{StO}_{2}\right)$ can be determined as

$$
\mathrm{StO}_{2}=C_{\text {oxy }} /\left(C_{\text {oxy }}+C_{\text {deoxy }}\right)
$$

For the example provided in Fig. 9, the estimated concentrations are $C_{\text {oxy }}=0.0063 \mathrm{mMoles}$ and $C_{\text {deoxy }}=0.0038 \mathrm{mMoles}$ with an oxygen saturation $\mathrm{StO}_{2}=0.62 \%$.

\section{CONCLUSION}

A micromachined sensor probe has been developed and tested for noninvasive oblique incidence diffuse reflectance spectrometry. The device miniaturization and fabrication precision provided by micromachining ensure reliable and repeatable high performance of the probe. The preliminary results indicate that it is reliable for use to estimate the optical properties and physiological information of skin. This information can potentially be used to assist the photodynamic therapy, and in vivo and noninvasive diagnosis of human skin pathologies.

\section{REFERENCES}

[1] V. P. Wallace, J. C. Bamber, D. C. Crawford, R. J. Ott, and P. S. Mortimer, "Classification of reflectance spectra from pigmented skin lesions, a comparison of multivariate discriminant analysis and artificial neural networks," Phys. Med. Biol., vol. 45, pp. 2859-2871, Oct. 2000.

[2] L. M. McIntosh, R. Summers, M. Jackson, H. H. Mantsch, J. R. Mansfield, M. Howlett, N. Crowson, and J. W. Toole, "Towards non-invasive screening of skin lesions by near-infrared spectroscopy," J. Invest. Dermatol., vol. 6, pp. 75-87, Jan. 2001.

[3] S. Sigurdsson, P. A. Philipsen, L. K. Hansen, J. Larsen, M. Gniadecka, and H. C. Wulf, "Detection of skin cancer by classification of Raman spectra," IEEE Trans. Biomed. Eng., vol. 51, no. 10, pp. 1784-1793, Oct. 2004.

[4] M. Gniadecka, P. A. Philipsen, S. Sigurdsson, S. Wessel, O. F. Nielsen, D. H. Christensen, J. Hercogova, K. Rossen, H. K. Thomsen, R. Gniadecki, L. K. Hansen, and H. C. Wulf, "Melanoma diagnosis by Raman spectroscopy and neural networks: Structure alterations in proteins and lipids in intact cancer tissue," J. Invest. Dermatol., vol. 122, pp. 443-449, Feb. 2004.

[5] T. L. Troy, D. L. Page, and E. M. Sevick-Muraca, "Optical properties of normal and diseased breast tissues: Prognosis for optical mammography," J. Biomed. Opt., vol. 1, pp. 342-355, Jul. 1996.

[6] P. R. Bargo, S. A. Pral, T. T. Goodell, R. A. Sleven, G. Koval, G. Blair, and S. L. Jacques, "In vivo determination of optical properties of normal and tumor tissue with white light reflectance and an empirical light transport model during endoscopy," J. Biomed. Opt., vol. 10, May 2005, 034018.

[7] R. A. His, D. I. Rosenthal, and E. Glatstein, "Photodynamic therapy in the treatment of cancer: Current state of the art," Drugs, vol. 57, pp. 725-734, May 1999. 
[8] H.-W Wang, M. E. Putt, M. J. Emanuele, D. B. Shin, E. Glatstein, A. G. Yodh, and T. M. Busch, "Treatment-induced changes in tumor oxygenation predict photodynamic therapy outcome," Cancer Res., vol. 64, pp. 7553-7561, Oct. 2004.

[9] V. Backman, M. B. Wallace, L. T. Perelman, J. T. Arendt, R. Gurjar, M. G. Muller, Q. Zhang, G. Zonios, E. Kline, T. McGillican, S. Shapshay, T. Valdez, K. Badizadegan, J. M. Crawford, M. Fitzmaurice, S. Kabani, H. S. Levin, M. Seiler, R. R. Dasari, I. Itzkan, J. Van Dam, and M. S. Feld, "Detection of preinvasive cancer cells," Nature, vol. 406, pp. 35-36, 2000.

[10] D. Hidovic-Rowe and E. Claridge, "Modelling and validation of spectral reflectance for the colon," Phys. Med. Biol., vol. 50, pp. 1071-1093, Feb. 2005

[11] L. T. Perelman, V. Backman, M. Wallace, G. Zonios, R. Manoharan, A. Nusrat, S. Shields, M. Seiler, C. Lima, T. Hamano, I. Itzkan, J. Vandam, J. M. Crawford, and M. S. Feld, "Observation of periodic fine structure in reflectance from biological tissue-A new technique for measuring nuclear size distribution," Phys. Rev. Lett., vol. 80, pp. 627-630, 1998.

[12] A. Garcia-Uribe, N. Kehtarnavaz, G. Marquez, V. Prieto, M. Duvic, and L. V. Wang, "Skin cancer detection by spectroscopic oblique-incidence reflectometry: Classification and physiological origins," Appl. Opt., vol. 43, pp. 2643-2650, 2004.

[13] L. H. Wang and S. L. Jacques, "Use of a laser beam with an oblique angle of incidence to measure the reduced scattering coefficient of a turbid medium," Appl. Opt., vol. 34, pp. 2362-2366, May 1995.

[14] S.-P. Lin, L. Wang, S. L. Jacques, and F. K. Tittel, "Measurement of tissue optical properties by the use of oblique-incidence optical fiber reflectometry," Appl. Opt., vol. 36, pp. 136-143, Jan. 1997.

[15] L. V. Wang and H. Wu, Biomedical Optics: Principles and Imaging. New York: Wiley-Interscience, 2007.

[16] H. C. Van de Hulst, Light Scattering by Small Particles. New York: Dover, 1981.

[17] S. Prahl, "Optical properties spectra," Oregon Medical Laser Center, Portland, OR, 2003 [Online]. Available: http://www.omlc.ogi.edu/ spectra

[18] M. Larsson, H. Nilsson, and T. Stromberg, "In vivo determination of local skin optical properties and photon path length by use of spatially resolved diffuse reflectance with applications in laser Doppler flowmetry," Appl. Opt., vol. 42, pp. 125-134, Jan. 2003.

[19] A. N. Bashkatov, E. A. Genina, V. I. Kochubey, and V. V. Tuchin, "Optical properties of human skin, subcutaneous and mucous tissues in the wavelength range from 400 to $2000 \mathrm{~nm}$, , J. Phys. D: Appl. Phys., vol. 38, pp. 2543-2555, Aug. 2005.

[20] C. R. Simpson, M. Kohl, M. Essenpreis, and M. Cope, "Near-infrared optical properties of ex vivo human skin and subcutaneous tissues measured using the Monte Carlo inversion technique," Phys. Med. Biol., vol. 43, pp. 2465-2478, Sep. 1998.

[21] J. S. Dam, C. B. Pedersen, T. Dalgaard, P. E. Fabricius, P. Aruna, and S. Andersson-Engels, "Fiber-optic probe for noninvasive real-time determination of tissue optical properties at multiple wavelengths," Appl. Opt., vol. 40, pp. 1155-1164, March 2001.

[22] H. Liu, D. A. Boas, Y. Zhang, A. G. Yodh, and B. Chance, "Determination of optical properties and blood oxygenation in tissue using continuous NIR light," Phys. Med. Biol., vol. 40, pp. 1983-1993, Nov. 1995.

Alejandro Garcia-Uribe was born in Mexico, in 1976. He received the M.S. degree from the Department of Electrical Engineering, Texas A\&M University, College Station, in 2002, where he is currently working towards the Ph.D. degree at the Department of Electrical and Computer Engineering.

His research interests are signal and image processing, microsensors and microfabrication, biomedical optics, and pattern recognition.
Karthik Chinna Balareddy received the B.S. degree in electrical engineering from Texas A\&M University, College Station, in 2002, where he is currently working towards the M.S. degree in electrical engineering.

His research interests lie in microfabrication of sensor probes.

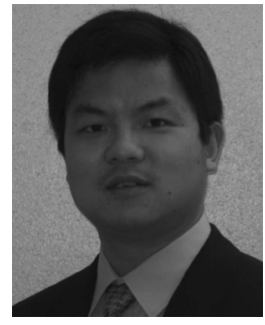

Jun Zou (S'98-M'02) received the Ph.D. degree in electrical engineering from the University of Illinois at Urbana-Champaign (UIUC), Urbana, in 2002.

From 2002 to 2004, he was a Postdoctoral Researcher in the Micro and Nanotechnology Laboratory, UIUC. In August 2004, he joined the Department of Electrical and Computer Engineering, Texas A\&M University, College Station, where he is currently an Assistant Professor. His research interests lie in the development of micro- and nanooptoelectromechanical devices and systems as well as novel micro- and nanofabrication methods. Since 1994, he has been conducting interdisciplinary research in this area and successfully developed a number of micromachined devices for applications in fluidics, acoustics, RF integrated circuits, and nanotechnology. His recent research effort is primarily focused on micro- and nanodiagnostic and surgical tools for biomedical applications. He has contributed more than 60 peer-reviewed journal and conference publications and holds three U.S. patents.

Dr. Zou is a member of the IEEE Electron Devices Society.

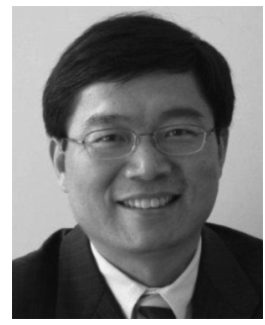

Lihong V. Wang (M'96-SM'00-F'06) received the $\mathrm{Ph} . \mathrm{D}$. degree in electrical engineering from Rice University, Houston, TX, in 1992.

$\mathrm{He}$ is currently the Gene K. Beare Distinguished Professor in the Department of Biomedical Engineering, Washington University, St. Louis, MO, where he is also the Director of the Optical Imaging Laboratory. He is the author or coauthor of more than 135 peer-reviewed journal articles published in various international journals. He is the author of the first textbooks in biomedical optics entitled Biomed ical Optics: Principles and Imaging (New York: Wiley-Interscience, 2007) He invented or discovered the dark-field confocal photoacoustic microscopy, photoacoustic Doppler sensing, focused scanning microwave-induced thermoacoustic tomography, exact reconstruction algorithms for photoacoustic or thermoacoustic tomography, frequency-swept ultrasound-modulated optical tomography, sonoluminescence tomography, Mueller-matrix optical coherence tomography, and oblique-incidence reflectometry. His Monte Carlo model of photon transport in scattering media has been used worldwide as a standard tool.

Dr. Wang is the Chair of the International Biomedical Optics Society. He is the recipient of the National Institutes of Health (NIH) FIRST Award, National Science Foundation (NSF) CAREER Award, and the Outstanding Young Scientist Award sponsored by the Johnson \& Johnson Medical, Inc., and the Houston Society for Engineering in Medicine and Biology. He is also a Fellow of the American Institute for Medical and Biological Engineering, the Optical Society of America, and the Society of Photo-Optical Instrumentation Engineers. He serves on the editorial boards for the Journal of Biomedical Optics and the Applied Optics. 